\title{
PRODUCCIÓN COMPARATIVA DE PALMITO ENTRE CUATRO VARIEDADES DE PEJIBAYE (Bactris gasipaes Kunth) ${ }^{1}$
}

\author{
Carlos Arroyo ${ }^{2}$, Jorge Mora ${ }^{3}$
}

\begin{abstract}
RESUMEN
Producción comparativa de palmito entre cuatro variedades de pejibaye (Bactris gasipaes Kunth). Se evaluó el rendimiento agroindustrial en cuatro variedades de pejibaye para palmito a las edades de 11,12 y 13 meses. Tres de ellas sin espinas (Diamantes-1, Diamantes-10, Diamantes20) y una con espinas (Utilis-Tucurrique). El ensayo se efectuó en la localidad de Guápiles, Costa Rica. Las variables estudiadas fueron: edad al inicio de la cosecha o precocidad; número de palmitos cosechados; longitudes de las vainas de la hoja previa a la hoja guía y de la hoja guía; longitud del pecíolo y de la lámina; longitud del palmito foliar y caulinar; pesos del palmito foliar, caulinar y total; diámetros del palmito foliar y caulinar; y rendimiento industrial (cajas/ha). Las variedades Diamantes-10 y Diamantes-20 fueron superiores en precocidad y número de palmitos cosechados. A los 12 meses el número de palmitos cosechados por hectárea fue de $7.067,4.493,4.381$ y 2.370 en las variedades Diamantes-10, Diamantes-20, Diamantes-1 y Utilis-Tucurrique, respectivamente. Estos resultados se utilizaron para evaluar los parámetros de producción industrial, los cuales presentaron diferencias $(\mathrm{P} \geq 0,0001)$ entre las variedades. El rendimiento promedio de palmito foliar y caulinar por tallo, fue de $226,67 \mathrm{~g}$ y $397,57 \mathrm{~g}$ por tallo en Diamantes-20; 208,91g y $379,04 \mathrm{~g}$ en Diamantes-10; $169,47 \mathrm{~g}$ y $264,62 \mathrm{~g}$ por tallo en Diamantes-1; y 124,81g y 122,01g por tallo en Utilis-Tucurrique. Además, las variedades sin espinas Diamantes-10 y Diamantes-20, produjeron un notorio mayor número de palmitos/ha y cajas/ha que la variedad Utilis-Tucurrique con espinas. Particular importancia reviste la gran diferencia obtenida en la producción de palmito caulinar, habiéndose mostrado las variedades sin espinas muy superiores a la variedad con espinas Utilis-Tucurrique.
\end{abstract}

\begin{abstract}
Comparative palm heart production among four varieties of peach palm (Bactris gasipaes Kunth). Palm heart production was evaluated at the ages of 11, 12 and 13 months in four varieties. Three of those varieties were thornless (Diamantes-1, Diamantes-10 and Diamantes-20) the other one had thorns in the trunk (Utilis-Tucurrique). The trial was conducted in Guapiles, Costa Rica. The parameters evaluated were: earlinesss; number of stems harvested; sheath lengths of the leaf previous-to-the emerging-leaf and of the emerging leaf; lengths of the petiole and leaf lamina; lengths of the foliar and caulinar heart of palm; weights of the foliar and caulinar hearts of palm; diameters of the foliar and caulinar heart of palm; and industrial yield (boxes/ha). The varieties Diamantes-10 and Diamantes-20 were superior in earliness and number of stems harvested. At the age of 12 months the number of stems harvested per hectare were: 7067, 4493, 4381 and 2370 respectively for the varieties Diamantes-10, Diamantes-20, Diamantes-1 and UtilisTucurrique. The average weight per stem of foliar and caulinar heart of palm respectively was $226.67 \mathrm{~g}$ and $397.57 \mathrm{~g}$ per stem for Diamantes-20; 208,91g and 379.04g for Diamantes-10; $169.47 \mathrm{~g}$ and 264.62g for Diamantes-1; and $124.81 \mathrm{~g}$ and $122.01 \mathrm{~g}$ for Utilis-Tucurrique. The thornless varieties produced a much larger caulinar heart of palm than the thorny variety, mainly Diamantes-20 and Diamantes- 10 .
\end{abstract}

\section{INTRODUCCIÓN}

En la actualidad el palmito de pejibaye es uno de los cultivos no tradicionales en Costa Rica. Su siembra se incrementó abruptamente entre los años 94 - 98. En 1998 el área sembrada aproximada fue de 12.718 hectáreas (Quirós 1999), por lo que fue considerado como uno de los cultivos de exportación más importantes.

\footnotetext{
${ }^{1}$ Recibido para publicación en marzo del 2002. Proyecto financiado por FITTACORI

2 Escuela de Zootecnia, Universidad de Costa Rica

${ }^{3}$ Escuela de Biología, Universidad de Costa Rica
} 
En el año 2001 se exportaron 10.992 toneladas métricas de palmito procesado, con un valor de 18,4 millones de dólares (Quirós 2002), producidas por grandes, medianos y pequeños productores.

Mora Urpí (1989) manifiesta que las siembras comerciales en Costa Rica, se ha utilizado una variedad con espinas, debido principalmente al bajo costo y la gran disponibilidad de semilla de ese material. Sin embargo, en países como Bolivia, Brasil, Ecuador y Perú, los materiales sin espinas (principalmente del cultivar Yurimaguas) son los que prevalecen. Con el propósito de evaluar materiales sin espinas, Mora et al. (1999), realizaron una prueba preliminar para comparar la producción de palmito entre nueve cultivares de pejibaye. Ellos obtuvieron una producción superior con los cultivares sin espinas sobre aquella de Utilis-Tucurrique, que es el cultivar con espinas que predomina en las plantaciones de Costa Rica. Esto no significa necesariamente que la presencia de espinas en el tallo esté correlacionado con la productividad, ésta puede ser debida a otros factores genéticos, pero la presencia de espinas si tiene importancia por cuanto es causa frecuente de accidentes de trabajo, obstaculiza las labores agronómicas y de industrialización.

Mora (1999a), manifiesta que, para lograr competitividad en el mercado mundial en los próximos años, el mejoramiento genético del cultivo del palmito debe orientarse hacia la obtención de materiales con las siguientes características: ausencia de espinas, crecimiento rápido, gran longitud de entrenudos, vaina muy larga, gran diámetro del tallo, fronda corta, lignificación lenta, mínimo tres internudos apicales suaves (palmito caulinar), resistencia general a enfermedades, alto rendimiento por palmito y alto rendimiento por hectárea $(3.000 \mathrm{~kg} / \mathrm{ha})$. El objetivo de este trabajo fue determinar las diferencias de producción entre cuatro variedades de pejibaye, tres de ellas nuevas sin espinas y una tradicional con espinas, al cabo de los primeros 13 meses de crecimiento en el campo.

\section{MATERIALES Y MÉTODOS}

\section{Localización del área experimental}

El ensayo se realizó en el área experimental de pejibaye que mantiene la Universidad de Costa Rica bajo el convenio MAG-UCR, en la Estación Experimental Los Diamantes, ubicada en Guápiles, cantón Pococí, provincia de Limón. La posición geográfica es $10^{\circ}, 13^{\text {' }}$ latitud norte y $86^{\circ}, 46^{\prime}$ longitud oeste, y la altitud es de $249 \mathrm{msnm}$. La prueba se llevó a cabo entre los meses de junio del 2000 a julio del 2001, con una temperatura promedio de $25,9^{\circ} \mathrm{C}$, una mínima de $16{ }^{\circ} \mathrm{C}$ en el mes de abril y la máxima de $35,2^{\circ} \mathrm{C}$ en el mes de setiembre; la precipitación acumulada fue de $4.065 \mathrm{~mm}$, con un promedio mensual de $338 \mathrm{~mm}$.

\section{Material experimental}

Para los tratamientos se utilizaron plántulas pequeñas con sólo dos hojas, de cuatro variedades de pejibaye para palmito: tres sin espinas, Diamantes-1, (de ascendencia Utilis-Guatuso), Diamantes-10 (de ascendencia Yurimaguas) y Diamantes-20 (de ascendencia Tuira-Darién); y una con espinas (Utilis-Tucurrique). El tiempo para alcanzar este estado de desarrollo en el almácigo no está considerado dentro de los 13 meses de desarrollo en el campo que se discuten aquí, porque es considerado el período de germinación de las semillas.

El diseño experimental que se utilizó, fue un irrestricto al azar con cuatro tratamientos y cinco repeticiones, para una densidad de siembra de 10.000 plantas por hectárea.

La parcela útil incluía 45 plantas dispuestas a 2,0 m de distancia entre hileras y $0,50 \mathrm{~m}$ entre plantas, para un total de 225 plantas útiles por tratamiento.

La fertilización inicial se efectuó con fosfato diamónico (DAP), y luego se aplicó mensualmente, en forma alterna, nitrato de amonio y la fórmula $18 \mathrm{~N}-5 \mathrm{P}-$ $15 \mathrm{~K}-6 \mathrm{Mg}-2 \mathrm{~B}-2 \mathrm{~S}$. El combate de malezas se realizó en forma integral con glifosato y 2,4-D y en ocasiones se chapearon las parcelas con motoguadaña.

\section{Variables evaluadas}

La evaluación se inició con la primera corta de palmito, los cuales eran cosechados cuando alcanzaban o superaban nueve centímetros de diámetro del tallo en su base, medidos a 20 centímetros de altura sobre el suelo. Las siguientes fueron las variables evaluadas:

Edad a inicio de cosecha o precocidad

Número de palmitos cosechados

Número de cajas estándar por hectárea

Longitud de la hoja previa a la hoja guía (vaina+ pecíolo+lámina)

Longitud total de la hoja guía

Longitud de la vaina de la hoja guía

Longitud del pecíolo de la hoja guía

Longitud de la lámina de la hoja guía

Longitud del palmito foliar (corazón de palmito o palmito industrial).

Longitud del palmito caulinar

Diámetro de la planta 
Diámetro del palmito foliar

Diámetro del palmito caulinar

Grado de apertura de la hoja guía (la longitud de la

hoja guía expuesta que estaba abierta)

Peso del palmito foliar

Peso del palmito caulinar

Peso total del palmito

Producción de palmito foliar por hectárea

Producción de palmito caulinar por hectárea

En las Figuras 1 y 2 se pueden apreciar algunas de las variables que se evaluaron. Se utilizaron las normas que utilizan las industrias para evaluar el rendimiento industrial (Ugalde 1999). Debe tenerse presente, en toda prueba comparativa de producción, que el "primer año de cosecha" se considera el tiempo comprendido entre la primera corta y los 12 meses siguientes. Por esta razón, aquí sólo se abarca el inicio de dicho primer año de cosecha, esto es, tres meses de cosecha. Se entiende aquí por "palmito foliar" la sección del palmito formado principalmente por las vainas de las hojas y en menor grado por otras secciones de las hojas (Figura 2). Este término se introduce en sustitución del anteriormente denominado "palmito industrial" o "corazón de palmito", ya que estos dos últimos términos resultan ambiguos. Por cuanto el "palmito caulinar", que es el palmito obtenido de la parte apical, suave y sólida del tallo, es también industrial y es "corazón".

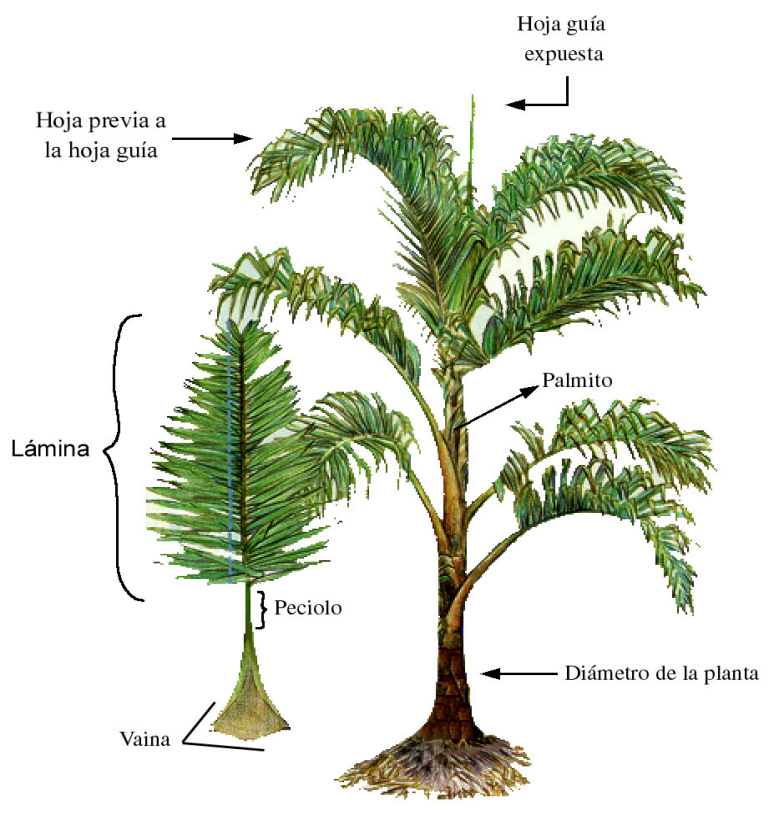

Figura 1. Detalle de la planta de palmito (modificado de MoraUrpí 1999). Estación Experimental Los Diamantes. Guápiles, Pococí, Limón. 2001.

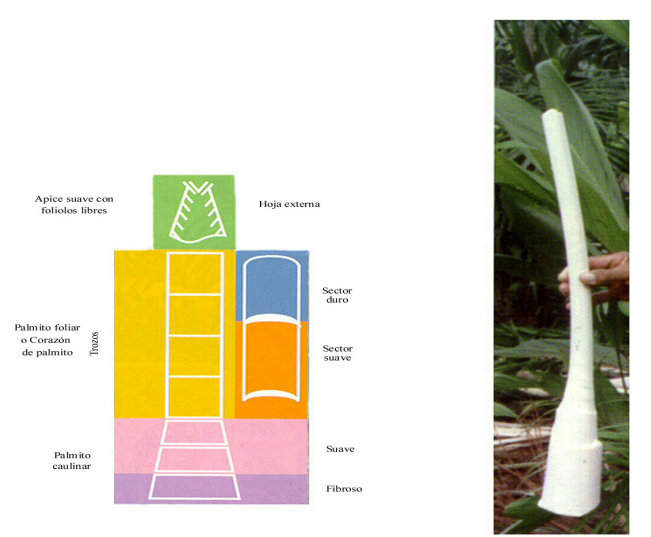

Figura 2. Palmito industrial (modificado de Mora-Urpí, 1999b). Estación Experimental Los Diamantes. Guápiles, Pococí, Limón. 2001.

\section{Análisis estadístico}

Se hizo un análisis de varianza y se obtuvieron las correlaciones y regresiones, utilizando el paquete de análisis estadístico SAS (1998). Las comparaciones de medias se realizaron utilizando la prueba Waller-Duncan, con una significancia P $(\alpha \leq 0,05)$. Para los gráficos se utilizó la hoja electrónica Excel.

\section{RESULTADOS Y DISCUSIÓN}

La primera operación de cosecha de palmito se realizó a los 11 meses después de la siembra en el campo, cuando el diámetro basal del tallo, medía como mínimo nueve centímetros. A esa edad se cosechó el 32,2\% de las cepas en la variedad Diamantes-10, el 26,1\% de Diamantes-20, el 14,3\% de Diamantes-1 y el 11,9\% de Utilis-Tucurrique. La conversión a producción promedio de palmitos por hectárea es de 3.221, 2.609, 1.429 y 1.185 respectivamente (Cuadro 1). Esta producción supera considerablemente lo informado por Reyes y Rankin (2000) en Bolivia, Colombia y la amazonía en Perú. Los resultados confirman la precocidad y productividad especialmente de la variedad Diamantes-10.

La segunda operación de cosecha se realizó a los 12 meses de la siembra en el campo o sea un año después de la siembra. Al completar este primer año de vida en el campo, la variedad Diamantes-10, se comportó vigorosa, precoz y muy productiva. Superó en producción acumulada de palmito foliar a Diamantes20 en un $57 \%$, a Diamantes- 1 en $61 \%$ y a Utilis-Tucurrique en $298 \%$, como se aprecia en el Cuadro 2. 
Cuadro 1. Producción promedio de palmitos y su rendimiento foliar y caulinar evaluados a los 12 meses de plantados en el campo. Estación Experimental Los Diamantes. Guápiles, Pococí, Limón. 2001.

\begin{tabular}{lccccc}
\hline Variedad & $\begin{array}{c}\text { Palmitos } \\
\text { cosechados/ha }\end{array}$ & $\begin{array}{c}\text { \% Cepas } \\
\text { cosechadas }\end{array}$ & $\begin{array}{c}\text { Palmito foliar } \\
\mathbf{k g} / \mathbf{h a}\end{array}$ & $\begin{array}{c}\text { Palmito caulinar } \\
\mathbf{k g} / \mathbf{h a}\end{array}$ & $\begin{array}{c}\text { Peso total } \\
\text { del palmito } \mathbf{~ k g} / \mathbf{h a} \\
\text { Foliar + caulinar }\end{array}$ \\
\hline Diamantes- 10 & $7067 \mathrm{a}^{*}$ & $70,7 \mathrm{a}$ & $1476.37 \mathrm{a}$ & $2678.68 \mathrm{a}$ & $4.155,05 \mathrm{a}$ \\
Diamantes- 20 & $4493 \mathrm{~b}$ & $44,9 \mathrm{~b}$ & $1018.43 \mathrm{~b}$ & $1786.28 \mathrm{~b}$ & $2.804,71 \mathrm{~b}$ \\
Diamantes -1 & $4381 \mathrm{~b}$ & $43,8 \mathrm{~b}$ & $742.45 \mathrm{c}$ & $1159.30 \mathrm{c}$ & $1.901,75 \mathrm{c}$ \\
Utilis-Tucurrique & $2370 \mathrm{c}$ & $23,7 \mathrm{c}$ & $295.80 \mathrm{~d}$ & $289.16 \mathrm{~d}$ & $584,96 \mathrm{~d}$ \\
\hline
\end{tabular}

* a,b,c,d= Valores medios con letra distinta en cada columna son estadísticamente diferetes, Duncan $(\mathrm{P} \leq 0,05)$

A los 13 meses de edad se efectuó la tercera y última corta aquí considerada. Totalizó el $85,6 \%$ de las cepas de la variedad Diamantes-10, 69,5\% de Diamantes1, 64,3\% de Diamantes- 20 y 46\% de Utilis-Tucurrique. Esto representa una producción promedio de palmitos por hectárea, hasta esa edad, de 8.560, 6.954, 6.430 y 4.597 respectivamente. Diferencias que son estadísticamente significativas entre todas ellas.

Estos resultados confirman los obtenidos por Mora et al. (1999), en una prueba comparativa de producción de palmito entre nueve cultivares de pejibaye en el cantón de Pococí, durante los cinco años que se extendió dicha prueba.

Los datos sobre la edad al inicio de la cosecha o precocidad, la cual es un parámetro sumamente impor- tante para evaluar el comportamiento productivo de las variedades, indican que la cosecha pudo haberse iniciado a los 10 meses en Diamantes-10 y Diamantes-20. Las variables: precocidad, número de palmitos cosechados en cada corta, longitud de la hoja previa a la hoja guía, longitud total de la hoja guía, y las longitudes de la vainas del pecíolo, de la lámina y de la hoja guía, de las variedades Diamantes-10 y Diamantes-20 fueron estadísticamente muy superiores a los promedios obtenidos por las variedades Diamantes-1 y Utilis-Tucurrique. Es importante indicar que esta última variedad mostró ser significativamente inferior a las otras tres variedades en todas las variables analizadas (Cuadro 3).

En lo concerniente al rendimiento industrial - esto es peso del palmito foliar y del palmito caulinar, la variedad Diamantes-20 se comportó ligeramente superior

Cuadro 2. Valores promedio de las variables estudiadas en las cuatro variedades. Estación Experimental Los Diamantes. Guápiles, Pococí, Limón. 2001.

\begin{tabular}{lcccc}
\hline \multicolumn{1}{c}{ Variables } & \multicolumn{4}{c}{ Variedades } \\
\cline { 2 - 5 } & Diamantes-20 & Diamantes-10 & Diamantes-1 & Utilis-Tucurrique \\
\hline Long hoja previa a hoja guía & $129,65 \mathrm{a} *$ & $119,89 \mathrm{~b}$ & $111,15 \mathrm{c}$ & $106,26 \mathrm{~d}$ \\
Longitud total hoja guía & $294,98 \mathrm{a}$ & $274,21 \mathrm{~b}$ & $274,21 \mathrm{~b}$ & $257,84 \mathrm{c}$ \\
Longitud vaina hoja guía & $57,76 \mathrm{a}$ & $52,01 \mathrm{a}$ & $39,36 \mathrm{~b}$ & $35,23 \mathrm{~b}$ \\
Longitud pecíolo hoja guía & $8,06 \mathrm{ab}$ & $6,30 \mathrm{~b}$ & $10,23 \mathrm{a}$ & $10,32 \mathrm{a}$ \\
Longitud de la lámina hoja guía & $241,41 \mathrm{a}$ & $229,02 \mathrm{bc}$ & $234,01 \mathrm{ab}$ & $224,40 \mathrm{c}$ \\
Peso total palmito & $624,24 \mathrm{a}$ & $587,08 \mathrm{a}$ & $434,09 \mathrm{~b}$ & $246,82 \mathrm{c}$ \\
Peso palmito foliar & $226,67 \mathrm{a}$ & $208,91 \mathrm{a}$ & $169,47 \mathrm{~b}$ & $124,81 \mathrm{c}$ \\
Peso palmito caulinar & $397,57 \mathrm{a}$ & $379,04 \mathrm{a}$ & $264,62 \mathrm{~b}$ & $122,01 \mathrm{c}$ \\
Diámetro palmito foliar & $2,50 \mathrm{a}$ & $2,46 \mathrm{a}$ & $2,47 \mathrm{a}$ & $2,52 \mathrm{a}$ \\
Diámetro palmito caulinar & $6,27 \mathrm{a}$ & $6,21 \mathrm{a}$ & $6,33 \mathrm{a}$ & $5,35 \mathrm{~b}$ \\
Longitud palmito foliar & $39,44 \mathrm{a}$ & $36,57 \mathrm{a}$ & $30,43 \mathrm{~b}$ & $22,15 \mathrm{c}$ \\
Longitud palmito caulinar & $15,02 \mathrm{a}$ & $14,48 \mathrm{a}$ & $10,47 \mathrm{~b}$ & $6,32 \mathrm{c}$ \\
Diámetro planta & $9,86 \mathrm{~b}$ & $10,07 \mathrm{a}$ & $9,78 \mathrm{~b}$ & $9,78 \mathrm{~b}$ \\
Apertura hoja guía & $73,68 \mathrm{a}$ & $81,68 \mathrm{a}$ & $72,09 \mathrm{ab}$ & $51,16 \mathrm{~b}$ \\
\hline
\end{tabular}

* a,b,c: Valores medios con letra distinta en sentido horizontal son estadísticamente diferentes, Duncan $(\mathrm{P}<0,05)$. 
a Diamantes-10, y ambas estadísticamente se comportaron muy superiores a Diamantes-1 y Utilis-Tucurrique. Esta última mostró un comportamiento muy pobre en lo referente al rendimiento, tanto de palmito foliar como caulinar, y obtuvo una producción $82 \%$ y $326 \%$ inferior respectivamente en comparación con Diamantes-20 (Figura 3 y Cuadro 1). Si se toma el peso unitario por palmito foliar y el número de palmitos cosechados por hectárea, se puede estimar el número de cajas estándar de palmito foliar obtenidas en la industria (Cuadro 3 y Figura 4), lo cual demuestra la notoria diferencia del rendimiento económico entre variedades a estas edades.

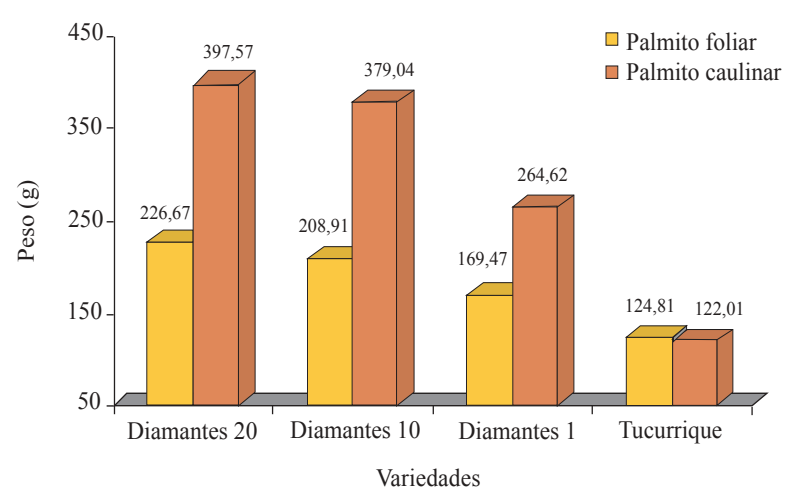

Figura 3. Peso promedio de palmito foliar y caulinar por tallo obtenido 12 meses después de la siembra en cuatro variedades de pejibaye. Estación Experimental Los Diamantes. Guápiles, Pococí, Limón. 2001.

La proporción del palmito total que representa el palmito caulinar en peso (Cuadro 2), fue de 63,68\% en la variedad Diamantes-20, 64,56\% en Diamantes-10, 60,96\% en Diamantes-1 y 49,43\% en Utilis-Tucurrique. Resalta un aspecto muy importante, en las tres variedades sin espinas, el rendimiento del palmito caulinar es considerablemente superior al del palmito foliar.

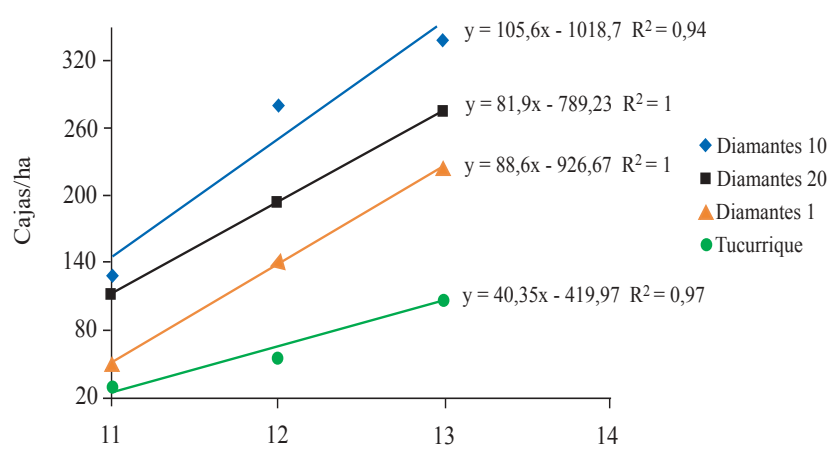

Figura 4. Estimación del número promedio de cajas de palmito foliar/ha a los 11,12 y 13 meses después de la siembra en cuatro variedades. Estación Experimental Los Diamantes. Guápiles, Pococí, Limón. 2001.

Este factor podría estar correlacionado con la ausencia de espinas, señalando que la presencia de espinas es un indicativo de mayor contenido de fibra en el tallo o mayor dureza de las fibras. Esta hipótesis, desde luego, requiere mayor investigación y confirmación.

El mayor peso del palmito caulinar le confiere, a estas variedades sin espinas, un importante valor adicional, ya que es de esperar que un mejor manejo industrial de este sector del palmito le confiera un mayor precio que el reconocido actualmente.

Al respecto, Ricardo Alfaro (Gerente de producción. DEMASA), manifesta que la empresa DEMASA la cual procesa el $80 \%$ de las exportaciones nacionales proyecta industrializar 39 millones de palmitos en el año 2001, de los cuales aprovechará únicamente el $40 \%$ del palmito caulinar de la variedad Utilis-Tucurrique en forma de mejillones de palmito.

Se conoce que existe una muy alta correlación entre la longitud de la vaina y la longitud del palmito foliar (Mora-Urpí y Arroyo 1999) por formar la vaina el tubo que lo conforma. Esta relación resulta obvia con

Cuadro 3. Rendimiento promedio acumulativo del palmito foliar - de cuatro variedades - por caja estándar (5,28 kg de palmito drenado) y por hectárea. Estación Experimental Los Diamantes. Guápiles, Pococí, Limón. 2001.

\begin{tabular}{|c|c|c|c|c|c|c|c|c|}
\hline \multirow[b]{2}{*}{$\begin{array}{l}\text { Variedad } \\
\text { g/palmito }\end{array}$} & \multicolumn{4}{|c|}{11 meses } & \multicolumn{2}{|c|}{12 meses } & \multicolumn{2}{|c|}{13 meses } \\
\hline & $\begin{array}{c}\text { palmito foliar } \\
n^{\circ}\end{array}$ & $\begin{array}{c}\text { palmitos/caja } \\
\mathbf{n}^{\circ}\end{array}$ & $\begin{array}{c}\text { palmitos/ha } \\
\mathbf{n}^{\circ}\end{array}$ & $\begin{array}{c}\text { cajas/ha } \\
\mathbf{n}^{\circ}\end{array}$ & $\begin{array}{c}\text { palmitos/ha } \\
n^{\circ}\end{array}$ & $\begin{array}{c}\text { cajas/ha } \\
\mathbf{n}^{\circ}\end{array}$ & $\begin{array}{c}\text { palmitos/ha } \\
\mathbf{n}^{\circ}\end{array}$ & cajas/ha \\
\hline Diamantes-10 & 208,91 & 25,27 & 3221 & 127,44 & 7067 & 279,62 & 8560 & 338,61 \\
\hline Diamantes-20 & 226,67 & 23,29 & 2609 & 112,00 & 4493 & 192,86 & 6430 & 275,82 \\
\hline Diamantes-1 & 169,47 & 31,16 & 1429 & 45,87 & 4381 & 140,62 & 6954 & 223,14 \\
\hline Utilis-Tucurrique & 124,81 & 42,30 & 1185 & 28,01 & 2370 & 56,02 & 4597 & 108,66 \\
\hline
\end{tabular}


los resultados aquí mostrados, en donde las dos variedades con mayor longitud de vaina - Diamantes-10 y Diamantes-20 - son significativamente superiores a Diamantes-1 y Utilis-Tucurrique en rendimiento unitario de palmito foliar, por tener estas últimas menor longitud de vaina (Cuadro 2).

Al considerar los resultados obtenidos de rendimiento agroindustrial (Cuadros 1, 2 y 3; y Figuras 3 y 4) se concluye que las variedades Diamantes-10 y Diamantes-20, presentan un gran potencial. Esto debido a que cumplen con una serie de características favorables que el cultivo del palmito debe innovar en un futuro próximo, tales como: ausencia de espinas, crecimiento rápido, mayor longitud en la vaina de la hoja guía, mayor diámetro del tallo, precocidad, mayor rendimiento por caja estándar (20 a 26 palmitos foliares para producir una caja de $5,2 \mathrm{~kg}$ de palmito drenado), un muy alto rendimiento de palmito caulinar, y una producción notoriamente superior de cajas por hectárea.

Debe resaltarse, que la ausencia de espinas reduce significativamente el costo de producción. Esto por cuanto disminuye o elimina los accidentes de trabajo provocados por la presencia de ellas en las variedades con espinas, lo cual representa un importante porcentaje de las incapacidades de los trabajadores. Al respecto, Víctor Arias (Gerente de Desarrollo Agrícola MAYVIC S.A. Horquetas, Sarapiquí) manifiesta que las incapacidades de los trabajadores por problemas de espinas es en promedio de 15 días por trabajador/ año, y que en casos extremos ha alcanzado hasta 140 días/año.

En forma complementaria a la adopción de estas nuevas variedades para elevar el rendimiento económico, la industria debe diversificar los procesos y presentaciones para lograr un mejor aprovechamiento del producto.

\section{LITERATURA CITADA}

MORA-URPÍ, J. 1989. Cultivares de pejibaye para palmito. Universidad de Costa Rica. Serie Técnica Pejibaye 1 (1): $8-9$.
MORA-URPÍ, J. 1999a. Origen, ecología y morfología del pejibaye. In: Palmito de pejibaye (Bactris gasipaes Kunth) su cultivo e industrialización. Eds. J. Mora-Urpí.; J. Gainza. San José, Costa Rica, Editorial Universidad de Costa Rica. pp.15-38.

MORA-URPÍ, J. 1999b. Bases para evaluar comercialmente futuras innovaciones en el cultivo. In: Palmito de pejibaye (Bactris gasipaes Kunth) su cultivo e industrialización. Eds. J. Mora-Urpí.; J. Gainza. San José, Costa Rica, Editorial Universidad de Costa Rica. pp.48-50.

MORA-URPÍ, J.; BOGANTES, A.; ARROYO, C. 1999. Cultivares de pejibaye para palmito. In: Palmito de pejibaye (Bactris gasipaes Kunth) su cultivo e industrialización. Eds. J. Mora-Urpí.; J. Gainza. San José, Costa Rica, Editorial Universidad de Costa Rica. pp.41-47.

MORA-URPÍ, J.; ARROYO, C. 1999. Cosecha de palmito. In: Palmito de pejibaye (Bactris gasipaes Kunth) su cultivo e industrialización. Eds. por J. Mora-Urpí.; J. Gainza. San José, Costa Rica, Editorial Universidad de Costa Rica. pp.118-121.

QUIRÓS, J. 1999. Exportaciones de Costa Rica. Dirección Mercadeo y Agroindustria. San José, Costa Rica. Consejo Nacional de Producción. Boletín Palmito 4(1): 1-7 p.

QUIRÓS, J. 2002. Exportaciones de Costa Rica. Dirección Mercadeo y Agroindustria. San José, Costa Rica. Consejo Nacional de Producción. Boletín Palmito $1(6): 1-7 p$.

REYES, C, R.; RANKIN, B, A. 2000. Cosecha y producción de palmito. In: El cultivo de chontaduro (Bactris gasipaes K) para palmito. Ed. R. Cuesta.; E. Peña.; J.Gómez. Tumaco, Colombia. Manual técnico $N^{\circ} 4$. Publicación de CORPOICA REGIONAL CINCO. pp.106 122.

UGALDE, S. J.C. 1999. Industrialización del palmito cultivado. In: Palmito de pejibaye (Bactris gasipaes Kunth) su cultivo e industrialización. Eds. J. Mora-Urpí.; J. Gainza. San José, Costa Rica, Editorial Universidad de Costa Rica. pp.180 - 190. 